\title{
LA NOCIÓN DEL TIEMPO EN LA CULTURA MAYA PREHISPÁNICA
}

\author{
The Notion of Time in the pre-Hispanic Maya Culture \\ Alexander Wolfgang Voss
}

Resumen: Se estudia la concepción del tiempo de los mayas prehispánicos mediante el análisis epigráfico y filológico de textos jeroglíficos, bajo los planteamientos teóricos de la escuela italiana de la historia de las religiones. Los datos indican que el tiempo es animado y formado por unidades discretas tangibles que se representan con un calendario de 365 días. Las características de cada día son fijadas por un oráculo de suertes de 260 unidades que es proyectado sobre el tiempo. Tanto el tiempo como el oráculo son pensados como dioses que se alternan en un orden fijo y cíclico y no como una magnitud física abstracta.

Palabras clave: tiempo, mayas, numeración, dioses, calendario, oráculo.

Abstract: The conception of time of the pre-Hispanic Maya is studied through epigraphic and philological analysis of hieroglyphic texts under the theoretical approaches of the Italian school of the history of religions. The data indicate that time is animated and consists of tangible discrete units that are represented by a calendar of 365 days. The properties of each day are provided by an oracle of lots of 260 units that is projected over time. Both time and the oracle are thought as gods that alternate in a fixed and cyclic order and not as an abstract physical quantity.

Keywords: time, Maya, numeration, gods, calendar, oracle.

Alexander Wolfgang Voss, doctor por la Universidad de Hamburgo, Alemania. Profesor-investigador de la Universidad de Quintana Roo, México. Temas de especialización: estudios de la cultura maya mediante la epigrafía, la etnohistoria y la arqueología, reconstrucción histórico-cultural de la sociedad y religión prehispánicas de las tierras bajas mayas. Correo electrónico: voss.uqroo@gmail.com.
Enviado a dictamen: 4 de febrero de 2015. Aprobación: 27 de abril de 2015.

Revisiones: 1. 


\section{Introducción}

L os elementos que más espacio ocupan en los textos jeroglíficos mayas de la época clásica son el Tzolkin, el Haab, la Cuenta Larga y los Números de Distancia. Estos registros epigráficos son interpretados por los investigadores de la cultura maya como datos cronológicos que se emplean para ubicar los acontecimientos, las personas y las localidades en una línea secuencial de tiempo (Lacadena, 2004: 83-84, 86-87; Rice, 2008: 281, 282-284). Siguiendo esta lógica, el Tzolkin y el Haab se entienden en su conjunto como fechas calendáricas, mientras que la cuenta larga y los Números de Distancia indican el tiempo transcurrido entre los diversos acontecimientos mencionados en las inscripciones. No obstante los resultados productivos que esta interpretación ofrece para los estudios de la cultura maya, estas informaciones no han sido evaluadas con base en los parámetros culturales de la sociedad maya prehispánica misma. Más bien se asume que estos registros provienen de un concepto físico o abstracto del tiempo, como lo construye la cultura occidental actual, sobre el cual se plasman luego interpretaciones mitológicas, históricas y proféticas (Lacadena, 2004; Rice, 2004: 56). Por ende, nos parece atractivo examinar los datos y las interpretaciones vigentes relativos al tiempo en la cultura maya prehispánica bajo los conceptos teóricos y metodológicos de la escuela italiana de la historia de las religiones (Massenzio, 2005; Mancini, 2007) y responder la sencilla pero importante cuestión de la concepción que los mayas prehispánicos mismos tenían del tiempo.

\section{La noción de tiempo y su contexto cultural}

Según el entendimiento científico derivado de la actual cultura occidental, el tiempo es una magnitud física que nos permite medir la duración de acontecimientos o la separación de estos en un orden secuencial. Estas secuencias nos permiten construir un pasado, un presente y un futuro para los eventos observados. Para un espectador que se encuentra dentro de un sistema de referencia, esto es, en un espacio con su propio estado de movimiento, el tiempo transcurre de manera uniforme. De ahí resulta que diferentes observadores pueden tener diferentes nociones de la duración y de la secuencia de los acontecimientos observados.

En la vida cotidiana, la existencia de tiempo se deduce de manera indirecta a partir de las variaciones perceptibles en los acontecimientos observados en el espacio. Estos pueden ser el movimiento constante de las manecillas de un reloj que nos marca segundos, minutos y horas, el recorrido del sol por el cielo con el que dividimos un día en sus segmentos -mañana, mediodía y tarde-, el cambio de día y noche, las estaciones del año, los ciclos de flora y fauna, la secuencia de nacimiento, vida y muerte o el registro de los días agrupados en semanas, meses y años mediante una cuenta sistematizada o calendario.

La noción que las sociedades humanas tienen de estos acontecimientos periódicos en el espacio es el resultado de su cultura. Existen dos conceptos básicos para interpretar estos sucesos en el espacio: cíclico y lineal. Según Farris (1987: 572), uno de estos es subordinado al otro (Rice, 2008: 293). En las sociedades que comparten la actual cultura de occidente, todos los hechos que ocurren en el tiempo son irreversibles, únicos y no repetibles, tienen un inicio y un fin. A este orden lineal de todos los eventos observados le llamamos cronología y lo representamos con una flecha o línea de tiempo. Esta noción es la base para la historia y la idea de desarrollo. Su fundamento está en el concepto de tiempo que es implementado por las religiones monoteístas, en particular el judaísmo y el cristianismo (Puech, 1958: 46-53).

Muy diferentes son aquellas sociedades cuya cultura se construye sobre la noción de que los acontecimientos específicos o las series de eventos recurrentes son el resultado de una repetición de estos mismos eventos después del transcurso de una determinada secuencia de sucesos. Este pensar en ciclos en que los acontecimientos tienen un orden inalterable, parte de la permanencia e inmutabilidad de los eventos en sí. Una vez establecido un hecho, éste se repite periódicamente dentro de una secuencia infinita de ciclos consecutivos. Nada se crea y nada se pierde, los cambios son más bien concebidos 
como manifestaciones de estos ciclos en que los acontecimientos se hacen, deshacen y rehacen de forma perpetua (Puech, 1958: 40-41). Aquí no encontramos el concepto de una historia basada en la singularidad de los acontecimientos ya que un ciclo carece de un punto de referencia que permite identificar el comienzo de los sucesos en el pasado, su desarrollo en el presente y su fin en el futuro. En culturas con pensamiento cíclico, la "historia de los acontecimientos", por así llamarla, implica siempre el estar después de un suceso y a la vez encontrarse nuevamente frente a él (Puech, 1958: 42-43). En sociedades con este tipo de pensamiento cíclico encontramos en la gran mayoría de los casos el mito que da legitimidad y fundamento a los acontecimientos y a las reglas sociales establecidas (Assmann y Assmann, 1998: 180-185; Malinowski, 1948).

Estas marcadas diferencias culturales respecto a la interpretación del tiempo están profundamente vinculadas a lo que en la cultura occidental denominamos religión (Brelich, 1977: 34-37). Es notable que las sociedades en cuyas culturas prevalece el entendimiento lineal del tiempo también son aquellas que han adoptado y forjado una religión que se sustenta en la creencia en un ser divino todopoderoso que transciende el mundo humano, existe fuera y sin él: el cristianismo en particular (Brelich, 1977: 51). En las religiones monoteístas de corte transcendental todo acontecimiento está ligado a la fe, la cual no es inteligible y para ella no hay evidencia tangible o intangible más allá de esa misma fe (Eliade, 1959: 109-110, 160).

En cambio, las culturas con una noción de tiempo cíclico tienden a poseer una religión inmanentemente cosmológica que es de manera categórica diferente a una religión de carácter transcendental (Zimmerman, 1963: 51; 1965: 141-142, 152-154). En una religión cosmológica todo acontecimiento está ligado a una causa tangible cuya relación causa-efecto es entendible y explicable con base en las observaciones del entorno natural y social. Cada fenómeno tiene un correlativo correspondiente en el mundo(Eliade, 1959: 98-99) y estemundo, con su respectivo orden, es divino o el reflejo de lo divino (Puech, 1958: 44).

Debido a su carácter inmanente y presencial en el mundo, la "religión cosmológica" abarca todo el universo y, por eso, todas las manifestaciones culturales y sociales. Materia prima, seres animados, las estructuras sociales, políticas y económicas, fenómenos naturales y el tiempo forman parte y son expresión de ese orden cósmico-divino. De ahí deviene que la categoría "religión" se vuelve absurda dentro de una cultura cosmológica ya que no se opone a ninguna otra categoría en esa cultura, a diferencia de la cultura cristiana-ilustrada de occidente, donde existe una separación del ámbito religioso-eclesiástico y del secular-cívico (Sabatucci, 1988: 44-48, 53-58). La variable más importante que permite identificar la religión en culturas cosmológicas es la creencia en la existencia de seres sobrehumanos o dioses (Spiro, 1966: 94) que corresponden a cada fenómeno natural, cultural y social en el mundo y a los que se les rinde culto, por lo que resulta oportuno hablar de una cultura politeísta, más que de una religión politeísta.

\section{El cómputo del tiempo y su ritual mántico en Mesoamérica}

Entrando en materia, es importante constatar que los mayas de la época precristiana poseyeron una cultura politeísta (Brelich 1960: 124-125, 133) y que compartían este rasgo cultural con todas las otras sociedades mesoamericanas, como lo declara Bartolomé de las Casas cuando escribe:

Toda esta tierra, de la que propiamente se dice la Nueva España, debía tener una religión y una manera de dioses, poco más o menos, y extendíase hasta las provincias de Nicaragua y Honduras, y volviendo hacia la de Xalisco, y llegaban, según creo, a la provincia de Colima y Culiacán [...] (De las Casas, 1967, I: 651, lib. III, cap. CXXIV).

El politeísmo es una característica de las culturas cosmológicas que piensan todas las fuerzas naturales y sociales como entidades sobrehumanas o dioses de quienes depende la existencia humana y que poseen poderes superiores al del individuo (Brelich, 1960: 127-128, 132; Brelich, 1977: 43-44, 48-49, 127; Spiro, 1966: 98). En este 
sentido, también el tiempo es una experiencia sensorial que debe considerarse una entidad sobrehumana o dios con su impacto inmediato en la existencia humana pero que se sustrae al control de las personas (Kremer, 2013: 295). En la cultura maya prehispánica es la presencia del sol o k'in, también conocido como Dios G o k'inich ajaw (Taube, 1992: 50-56; Thompson, 1971: 142), con su movimiento orbital por el cielo que marca visualmente el tiempo, específicamente el tiempo de un día. Nótese que k'in se refiere simultáneamente a día y a sol.

La importancia del tiempo y de sus dioses se hace evidente en las ceremonias y fiestas que los mayas prehispánicos realizaban en su honor a lo largo de un año (Landa, 1959: 60-103). El cómputo para esas fiestas anuales de los dioses se realizaba mediante un calendario - Haab en maya y $X_{\text {iuhpohualli en }}$ náhuatl- que registra 360 fechas distribuidas en dieciocho veintenas - los llamados meses-, más un grupo residual de cinco fechas al final de cada ciclo. Al igual que los dioses, este calendario de 365 unidades es un denominador común de todas las sociedades mesoamericanas, como lo demuestran Edmonson (1988) en su compilación de calendarios mesoamericanos y Kirchhoff en su análisis comparativo de las dieciocho fiestas anuales de Mesoamérica del Posclásico Tardío (Kirchhoff, 1971 y 2002).

El tercer elemento que caracteriza la unidad cultural mesoamericana en su conjunto es la cuenta de 260 unidades - Tzolkin en maya y Tonalpohualli en náhuatl- que, según Caso, existió por lo menos a partir del horizonte Preclásico Tardío (Caso, 1971: 199-200; Edmonson, 1988). Este ciclo adivinatorio fue creado a partir de la permutación de trece números y veinte signos o letras y recibe erradamente el nombre de "calendario ritual" debido a que no corresponde con el ciclo de las ceremonias o fiestas del año. Su carácter mántico no fue del todo entendido por los frailes misioneros ni por los primeros investigadores de las culturas mesoamericanas (Seler, 1900: I, 5). No obstante, para los mayistas la errónea interpretación del Tzolkin como calendario sigue formando parte de su entendimiento académico (Kremer, 2013: 298; Lacadena, 2004; Rice, 2004 y 2008; Thompson, 1971: 66-103). En este sentido vale recordar a Sahagún, quien escribe en el apéndice al libro cuatro de su Historia general que esta cuenta de los mexica era arte para adivinar la fortuna o ventura, y no un calendario:

Esta cuenta, muy perjudicial y muy supersticiosa y muy llena de idolatría, como parece en este libro cuarto, algunos la alaban mucho diciendo, que era muy ingeniosa y que ninguna mácula tenía; esto, dijeron por no entender a qué fin se endereza esta cuenta, el cual es muy malo, idolátrico. De poco entendieron la muchedumbre de supersticiones y fiestas, y sacrificios idolátricos que en ella se contienen, y llamaron a esta cuenta el calendario de los indios no entendiendo que esta cuenta no alcanza todo el año porque no tiene más de doscientos sesenta días de círculo, y luego torna a su principio y así no puede ser calendario y nunca lo fue, porque el calendario [...] contiene todos los días del año y las fiestas del año [...] (Sahagún, 1999: 256).

Líneas más abajo, Sahagún continúa diciendo que:

En lo que dice que en este calendario no hay cosa de idolatría es falsísima mentira, porque no es calendario sino arte adivinatoria, donde se contienen muchas cosas de idolatría, y muchas supersticiones y muchas invocaciones de los demonios, tácita y expresamente, como parece en todo este cuarto libro precedente [...] (Sahagún, 1999: 256).

Lo mismo aplica al Tzolkin, que en el libro de Chilam Balam de Kaua y en el Códice Pérez - Chilam Balam de Mani- se denomina u mutil u chuenil kin sansamal, "el destino y el arte de los días para todos los días", o u mutil uinic sansamal, "el destino del hombre todos los días", y se refiere a una característica que poseen los días y que es mutable y alterable según el caso (Barrera, 1975), pero no a un calendario.

El origen de este ritual mántico u oráculo de suertes, si utilizamos el término acuñado por la ciencia de las religiones para este tipo de arte adivinatoria, es considerado divino, tal como se desprende del mito 
etiológico que Gerónimo de Mendieta reproduce parcialmente en el capítulo XIV del libro segundo de su Historia eclesiástica indiana:

Para tractar de las fiestas que estos indios de la Nueva España (en especial los de México, Texcuco y Tlaxcala) hacían a sus dioses, es de saber cuanto a lo primero, que tenían su calendario por donde se regían, y tenían señalados sus días del año para cada uno de los diablos a quien hacían fiesta y celebraban, así como nosotros tenemos dedicado su día en tal o tal mes a cada uno de los santos. Que en esto parece haber tomado el maldito demonio oficio de mona, procurando que su babilónica e infernal iglesia o congregación de idólatras y engañados hombres, en los ritos de su idolatría y adoración diabólica remedase (en cuanto ser pudiese) el orden que para reconocer a su Dios y reverenciar a sus santos tiene en costumbre la Iglesia católica. Y dando relación los indios viejos del principio y fundamento que tuvo este su calendario, contaban una tonta ficción, como son las demás que creían cerca de sus dioses. Dicen que como sus dioses vieron haber ya hombre criado en el mundo y no tener libro por donde se rigiese, estando en tierra de Cuernavaca en cierta cueva dos personajes, marido y mujer, del número de los dioses, llamados por nombre él Oxomoco y ella Cipactonal, consultaron ambos a dos sobre esto. Y pareció a la vieja sería bien tomar consejo de su nieto Quetzalcoatl, que era el ídolo de Cholula [...], dándole parte de su propósito. Parecióle bien su deseo, y la causa justa y razonable: de manera que altercaron los tres sobre quién pondría la primera letra o signo del calendario. Y en fin, teniendo respeto a la vieja, acordaron de le dar la mano en lo dicho. La cual andando buscando qué pondría al principio del dicho calendario, topó en cierta cosa llamada Cipactli, que la pintan a manera de sierpe, y dicen andar en el agua, y que le hizo relación de su intento, rogándole tuviese por bien ser puesta y asentada por primera letra o signo del tal calendario; y consistiendo en ello, pintáronla y pusieron Ce Cipactli, que quiere decir "una sierpe". El marido de la vieja puso dos cañas, y el nieto tres casas etcétera, de esta manera fueron poniendo hasta trece signos en cada plana, en reverencia de los autores dichos y de otros dioses que en medio de cada plana tenían los indios, pintados y muy asentados en este libro del calendario, [...] (Mendieta, 2001: 210-211).

Según este relato, fueron los dioses -Cipactonal, Oxomoco y Quetzalcoatl- quienes escogieron sus signos del oráculo de suertes para que el hombre se gobernase por ellos, es decir, los signos son patrocinados por estos dioses y tienen, por lo tanto, un origen divino. A su vez, los dioses pueden ser identificados y representados por los números - en este caso 1, 2 y 3- que anteceden los tres signos - Cipactli (sierpe), Acatl (caña) y Calli (casa) - en el relato. Thompson, basándose en fuentes etnográficas, afirma que en la cultura mesoamericana los seres sobrehumanos pueden ser significados mediante números (Thompson, 1971: 12, 66, 69-70, 96, 103-104).

El mismo patrón se observa en los registros epigráficos mayas. Las variantes antropomorfas de los números son los dioses mismos. El número 'l' representa la cabeza de una mujer, el equivalente a Cipactonal, el número '2' es la cabeza de un hombre con un tocado en forma de puño, correspondiente a Oxomoco, y el número ' 3 ' es un joven con una diadema de flores y una orejera que contiene el signo i $k$ ' (viento), una de las características de Quetzalcoatl en su aspecto de dios del viento y de las tormentas (ver figura 1).

La proyección del Tzolkin sobre el Haab permite un total de 18980 combinaciones en 52 años a 365 días computados, que se conoce como "rueda calendárica". Debido a la aritmética que se genera entre ambos ciclos, el Tzolkin con sus 260 combinaciones y el Haab con sus 365 unidades, cada fecha del Haab puede tomar cuatro signos del Tzolkin, que a su vez se conectan con los trece números. De esta forma, cada posición del Haab posee 52 diferentes oráculos, trece variantes por cada uno de los cuatro signos del Tzolkin.

Para ilustrar el alegato de que el Tzolkin es un oráculo de suertes y no un calendario, comparamos el Códice de Dresde con el Tablero 2 de Laxtunich, un sitio arqueológico ubicado en el valle del Usumacinta, 
en Chiapas. En el registro b de las páginas 5 y 6 del mencionado códice se ven cuatro dioses taladrando objetos duros (hoch' u chich). Al margen izquierdo de este t'ol o "almanaque" está pintada una columna con los signos Manik', Kawak, Chuwen, Ak'bal y Men del Tzolkin. El número base para los cálculos con el cual se combinan los signos es el número 'l', que se representa mediante un punto rojo y se encuentra en la parte superior de la misma columna (ver figura 2). Aplicando la aritmética de este t'ol, vemos que el Tzolkin 1-Kawak coincide con la representación del llamado Dios Q del inframundo (Thompson 1971: 131; Taube 1992: 105-112) en el último cuadro (Thompson 1972: 38).

En el tablero de Laxtunich vemos a dos personajes sentados sobre las fauces del inframundo. El individuo de la izquierda, el gobernante Itzamnaj Balam IV de Yaxchilán, mencionado aquí con su apodo chan taj mo k'uh[ul] pachan ajaw, está igualmente taladrando un objeto mediante un palillo, y el otro individuo, a la derecha, es precisamente una entidad del inframundo llamada k'uh[ul]u chan ba way ch'ok sajal matawi[l]. La inscripción entre los dos personajes marca que se está haciendo fuego o humo (hoch' butz'/k'ak'). Este acontecimiento es antecedido por una Rueda Calendárica que consiste del Tzolkin 1-Kawak y el Haab 2 Wo (ver figura 3).

Se observa que hay una coincidencia entre las escenas y los textos de ambos documentos. El ritual mántico es igual en ambos casos con respecto a la actividad que se realiza: se taladran objetos en relación con el Tzolkin 1-Kawak. Si este paralelismo es meramente fortuito o se trata de la manifestación de un patrón cultural de larga duración —-del Clásico al Posclásico Tardío- deberá ser demostrado mediante un estudio sistemático cuantitativo. No obstante, es importante señalar que el Tzolkin anotado en el códice no tiene vínculo alguno con el Haab, lo que muestra así su carácter funcional independiente del calendario como oráculo, mientras que en el tablero el mismo ritual es fijado mediante el Haab en el tiempo, adhiriéndole con la información 2 Wo una fecha a un día específico.

Se afirma entonces lo que dice Sahagún, que este oráculo no es un dispositivo para medir el tiempo, más bien se trata de un ritual mántico plasmado en el tiempo que le permitía al politeísta mesoamericano el contacto con los dioses en cada uno de los días de un año para llegar a saber e influir sus cualidades con el objetivo de garantizar el bienestar de sus semejantes (Caso, 1971: 192-193; Thompson, 1971: 103; Kremer, 2007: 6-7; Kremer 2013: 650-654), lo que a la vez confería individualidad y carácter a los días. Este tipo de ritual mántico de suertes plasmado en el tiempo también es denominado oráculo calendárico.

\section{La noción de tiempo en la cultura maya del Clásico}

En el caso de la cultura maya clásica no tenemos información más allá de las inscripciones jeroglíficas para deducir la noción de tiempo que los mayas poseyeron en esa etapa de su desarrollo cultural. En ese periodo, el calendario de 365 días y el ritual mántico de 260 unidades se encontraban en plena operación. Adicionalmente a esa Rueda Calendárica, en el Clásico se efectuaba un cómputo de unidades llamado Cuenta Larga o también Serie Inicial que servía para registrar periodos de tiempo cuya base era un sistema vigesimal modificado (Kettunen y Helmke, 2011: 47). Aparentemente, esa Cuenta Larga genera una línea de tiempo, pero está constituida por ciclos donde los periodos pequeños se anidan dentro de las unidades superiores. La unidad más pequeña es el $k$ 'in (día), seguido por el winal (mes) de veinte días, el tun o haab (año) de 360 días, el k'atun de veinte tunes o 7200 días, el baktun de veinte k'atunes o 144000 días y ciclos superiores como el piktun, el k'alabtun y el k'inchiltun, todos múltiples de veinte (ver figura 4).

En este sistema no existe un punto de partida propiamente dicho. Cada ciclo tiene su propio ritmo y permite observar la repetición de acontecimientos específicos en diferentes periodicidades que siempre se identifican mediante el Tzolkin o una Rueda Calendárica. En este sentido, los mayas del Clásico usaban un evento singular para el registro del tiempo transcurrido, la creación del mundo de los seres humanos por los dioses. Este suceso específico es interpretado por los mayistas como punto de partida para la existencia de una noción lineal del tiempo entre los mayas precolombinos 
(Lacadena, 2004: 84; Rice 2008: 282-284). No obstante, ese punto de referencia sólo marca uno más de los hechos primordiales que forman parte del porvenir cíclico del tiempo, ya que para el caso de los mexica y maya k'iche' se documentan "creaciones" previas a esta última en que surge el ser humano.

Los mayas prehispánicos marcaban este punto de referencia con la Rueda Calendárica 4-Ajaw 8 Kumk'u y lo relacionaron con la Cuenta Larga, en la cual todas las unidades de $k$ 'in hasta baktun poseen el valor '0', lo que en la epigrafía maya se representa mediante la fórmula '0.0.0.0.0', donde cada unidad es separada de las otras mediante puntos. Del baktun, que ocupa el primer lugar a la izquierda, se procede sucesivamente hasta la última posición a la derecha, que representa la unidad k'in. Pero no hay que olvidar que los mayas eran conscientes de la existencia de ciclos de tiempo superiores que raras veces se registraban y sobre cuyos coeficientes no había unanimidad entre los escribas.

Los Números de Distancia emplean las mismas unidades de tiempo que la Cuenta Larga, pero el orden de los diferentes períodos de tiempo se invierte, por lo que queda la unidad más pequeña en la primera posición a la izquierda y las otras le siguen subsecuentemente. Una particularidad de estos Números de Distancia es el hecho de que en la mayoría de las inscripciones se suprime la representación de la unidad k’in. Su coeficiente es añadido a la próxima unidad o posición, el winal (ver figura 5).

A menudo, estos Números de Distancia son precedidos por jeroglíficos que escriben la forma verbal del vocablo tz'ak. Este verbo se refiere a la actividad de contar grados y escalones y otras cosas que van unas encima de otras, e introduce el dato sobre el tiempo transcurrido entre una Rueda Calendárica y otra. Deducimos a través de la semántica de tz'ak que el tiempo contabilizado se piensa en unidades discretas tangibles que pueden ser apiladas o que son puestas en un orden consecutivo. Esta información apoya la noción de una identidad singular para cada día que se genera a partir del oráculo calendárico del Tzolkin que se asocia con cada día.

Otro elemento que nos permite acercarnos a la noción del tiempo en las inscripciones mayas del
Clásico es el Clasificador Numeral que se inserta entre el coeficiente y las unidades del tiempo que son contadas. En las lenguas mayas, los Clasificadores Numerales son morfemas obligatorios que se combinan con sustantivos cuantificables para que estos puedan ser contabilizados (Suárez, 1995: 143-145). Mientras que el coeficiente tiene la función de cuantificar un objeto, el Clasificador Numeral indica la clase semántica a la cual pertenece el objeto. En su investigación cuantitativa realizada con 2500 informaciones calendáricas de la época Clásica, Prager (2003) identifica el morfema te como Clasificador Numeral en Cuentas Largas, Números de Distancia y el Haab (ver figura 6). El mismo Clasificador Numeral se sigue también usando en las fechas mayas anotadas en los libros de Chilam Balam.

Según Miram (1983), el maya yucateco moderno posee más de doscientos diferentes Clasificadores Numerales, muchos de los cuales también son documentados en los diccionarios coloniales y en las diversas cuentas de días de los textos jeroglíficos. Algunos de ellos son, por ejemplo: ban (partícula para contar montones), luk (andar paso a paso), pi' o pis (para contar años, meses, días, reales, pesos) y tuk (montón de cosas menudas ajuntadas, como sal, tierra, piedras, leña, etcétera), por mencionar sólo algunos.

Como se dijo, estos Clasificadores Numerales indican la clase semántica a la cual pertenece el objeto que se cuenta. En el caso de los Clasificadores Numerales identificados en las inscripciones jeroglíficas, todos se refieren a objetos discretos que pueden ser amontonados o puestos en una fila. Esto implica que el tiempo no era considerado un flujo continuo como lo marca la física moderna, sino la suma consecutiva de los días tal como ya vimos en el caso del verbo tz'ak. A cada k'in le es asignada una posición a través de las veintenas del Haab y la Cuenta Larga. Su individualidad, sin embargo, le es asignada mediante el Tzolkin.

Los Clasificadores Numerales también marcan la diferencia entre las unidades de tiempo y la información mántica. A diferencia de las unidades de tiempo, el Tzolkin nunca posee Clasificadores Numerales. En el Tzolkin los números del 1 al 13 se conectan de manera inmediata con el signo. Por lo tanto, el Tzolkin no representa 
unidades contabilizadas, sino un nombre compuesto por dos sustantivos: un dios y un signo patrocinado por un dios. Este nombre, así lo refiere Landa al tratar el Haab de la época posclásica maya, se suma a cada día del calendario de 365 días y le otorga su identidad particular y su valor mántico del oráculo de las suertes, como ya se mencionó arriba.

$\mathrm{Al}$ respecto vale mencionar que en los ritos y las narrativas mitológicas mesoamericanas muchos dioses poseen nombres formados al uso del Tzolkin, mediante un número y un signo. En el Popol Vuh de los maya k'iche' son, por ejemplo, Hunahpu (1-Ajpu), Hun Batz (1-Batz'), y los señores del inframundo, Hun Came (1Kame) y Vucub Came (7-Kame). En las fuentes aztecas, principalmente en el Códice Florentino de Sahagún, encontramos diversas referencias a seres sobrehumanos o dioses que se identifican mediante nombres del Tonalpohualli a la usanza del Tzolkin. Ce Ãcatl (1-Caña) es el sobrenombre de Quetzalcoatl en su aspecto de estrella del alba, Nãhui Ãcatl (4-Caña) es el nombre calendárico del fuego (Xiuhtéuctli), Nãhui Ehecatl (4-Viento) es el sobrenombre de los Tlaloques o dioses de la lluvia y Chicõme Cõatl (7-Serpiente) es la encarnación del sustento, en especial del maíz, por mencionar sólo algunos. Estas referencias son vivo testimonio de que los dioses proveían con sus propiedades al oráculo de las suertes, que a su vez impregnaban cada uno de los 365 días de su calendario con características específicas. Así, cada día quedaba bajo la influencia de sus poderes divinos que se alternaban en un orden fijo y cíclico representado por la Rueda Calendárica.

Este vínculo indisoluble entre los dioses, el oráculo calendárico y el tiempo se manifiesta de manera singular en dos inscripciones, en el Panel 2 del Edificio de la Serie Inicial de Xcalumkin y en el friso de 96 jeroglíficos del cuarto 29 sub de la Estructura 1 de Ekbalam. En el caso del friso de Ekbalam se mencionan dos fechas - ll-Eb 10 Sotz' y 8-Imix 19 Xul- que distan 49 días una de la otra. En vez de usar un Número de Distancia para conectar ambas fechas, el escriba registró uno por uno todos los 49 oráculos por su nombre -12 Ben hasta 8 Imix - (Lacadena, 2003) y resaltó de esta manera su importancia primordial para el transcurso del tiempo.
En el caso de la inscripción del panel de Xcalumkin se trata de una elaborada Serie Inicial con la Cuenta Larga 9.15.12.6.9 y la Rueda Calendárica 7-Muluk 1 K'ank'in. En las unidades tun y winal y el Haab se identifica el vocablo te como el Clasificador Numeral. Pero en la unidad k'in aparece como Clasificador Numeral la voz tak (ver figura 7). En maya yucateco tak es un sufijo plural para nombres, adverbios y participios, en ch'orti maya es un sufijo plural que va con algunos términos de seres humanos (Hull, 2005: 103) y en las inscripciones jeroglíficas este sufijo aparece exclusivamente con entidades animadas.

Este hecho permite inferir, primero, que el tiempo es considerado un ente animado (Rice, 2008: 285), es el dios sol o k'inich ajaw, que actúa por impulso propio $y$, segundo, es razonable pensar que son los dioses del Tzolkin que se suman a kinich ajaw con sus calidades mánticas. Esta triada de dioses es y hace el tiempo. Esto es lo que realmente explica la "obsesión" de los mayas por el registro del tiempo y su fijación en eventos recurrentes. El tiempo no es una magnitud física abstracta, sino el testimonio tangible de la presencia de dioses específicos en el espacio que permite al hombre mesoamericano enfrentar su fragilidad a través de los ciclos de los dioses que dominan el tiempo.

\section{Conclusiones}

La presente investigación partió del cuestionamiento de la noción que tenían los mayas prehispánicos del tiempo. Se analizaron los diversos elementos que constituyen el registro del tiempo en las inscripciones jeroglíficas de la cultura maya prehispánica profundizando en algunos aspectos ya conocidos, pero también incorporando elementos nuevos como los Clasificadores Numerales, que previamente no habían recibido la atención necesaria. Estos Clasificadores Numerales indican que el tiempo o k'in es una entidad animada (Rice, 2008: 285) y está representada por 365 unidades discretas tangibles que en su conjunto forman el Haab o calendario propiamente dicho.

Con fundamento en los planteamientos de la escuela italiana de historia de las religiones se explica que, en la mentalidad de una cultura politeísta como la maya prehispánica, todo fenómeno sensorial y perceptible 
como el tiempo, que impacta en la existencia del hombre pero que se sustrae a su influencia, es concebido como un ser sobrehumano o dios (Brelich, 1960: 127-128, 132) y no como una magnitud abstracta tal como se presenta en la literatura mayista sobre el tema (Lacadena, 2004; Rice, 2008).

También se demuestra que el Tzolkin con sus 260 unidades no puede ser un calendario ya que no contabiliza el tiempo con Clasificadores Numerales como el Haab, sino es un oráculo de suertes patrocinado por dioses que se extiende en el tiempo (Kremer, 2013: 295-297, 651).

La alternación de los dioses del tiempo y del oráculo en el orden fijado por la Rueda Calendárica indica que el tiempo en la cultura maya prehispánica era primeramente cíclico y el resultado de las actividades de los dioses en el espacio que comparten con el hombre. En este sentido, la Cuenta Larga del periodo clásico extiende el registro del tiempo cíclico más allá de la Rueda Calendárica y crea a los ojos del investigador occidental, con su mentalidad unilineal y progresiva, la sensación de una cuenta lineal para el registro del tiempo transcurrido mediante la fijación de un punto arbitrario que marca la creación del mundo en que vive el hombre.

La permanencia del calendario y del oráculo de suertes a lo largo de más de mil quinientos años da constancia de la continuidad y estabilidad cultural que rige a los mayas, y a la vez nos hace ver la gran importancia que poseen estos dispositivos para todas las sociedades mesoamericanas.

\section{Referencias bibliográficas}

Assmann, Aleida y Jan Assmann (1988), "Mythos". En Hubert Cancik et al. (eds.), Handbuch religionswissenschaftlicher Grundbegriffe, t. IV. W. Stuttgart: Kohlhammer, pp. 179-200.

Barrera Vázquez, Alfredo (1975 [1943]), Horóscopos mayas o el pronóstico de los 20 signos del Tzolkin, según los libros de Chilam Balam de Kaua y de Mani. Mérida, Yucatán: Área Maya/Mayan Area.

Brelich, Ángelo (1960), “Der Polytheismus". En Numen, núm. 7, pp. 123-136.
Brelich, Ángelo (1977), "Prolegómenos a una historia de las religiones". En Henri-Charles Puech (ed.), Las religiones antiguas 1 (Historia de las religiones 1). Madrid: Siglo XXI, pp. 30-97.

Caso, Alfonso (1971), “iReligión o religiones mesoamericanas?" En Verhandlungen des XXXVIII. Internationalen Amerikanistenkongresses, Stuttgart-München 12-18. August 1968. Munich: Renner, pp. 189-200.

Edmonson, Munro Sterling (1988), The Book of the Year. Middle American Calendrical Systems. Salt Lake City: University of Utah.

Eliade, Mircea (1959), Cosmos and History. The Myth of the Eternal Return. Nueva York: Harper \& Bros.

Farriss, Nancy (1987), "Remembering the Future, Anticipating the Past: History, Time and Cosmology among the Maya of Yucatan". En Comparative Studies in Society and History. vol. 29, núm. 3, pp. 566-593.

Hull, Kerry (2005), "An Abbreviated Dictionary of Ch'orti' Maya”, informe de investigación presentado a la Foundation for the Advancement of Mesoamerican Studies, Inc.. Disponible en http://www.famsi.org/reports [consultado el 1 de mayo de 2013].

Kettunen, Harri y Christopher Helmke (2011), Introducción a los jeroglificos mayas. XVI Conferencia Maya Europea Copenhague 2011. Copenhague: Universidad de Copenhague. Disponible en http://www.wayeb.org/ download/resources/wh20llspanish.pdf [consultado el 24 de enero de 2012].

Kirchhoff, Paul (1971), "Las 18 fiestas anuales de Mesoamérica: 6 fiestas sencillas y 6 fiestas dobles". En Verhandlungen des XXXVIII. Internationalen Amerikanistenkongresses, Stuttgart-München 12-18. August 1968. Munich: Renner, pp. 207-221.

Kirchhoff, Paul (2002), "Las 18 fiestas anuales en Mesoamérica: 6 fiestas sencillas y 6 fiestas dobles". En Carlos García Mora et al. (comp.), Kirchhoff, escritos selectos: Estudios mesoamericanistas, t. I. México: UNAM, pp. 401-415.

Kremer, H. Jürgen (2007), "Religión: una definición para Mesoamérica”. En Ketzalcalli, núm. 2, pp. 3-19.

Kremer, H. Jürgen (2013), Religion heisst Dienst an den Göttern: Eine kultur- und religionswissenschaftliche Untersuchung zu den Grundlagen des mesoamerikanischen 
Polytheismus. Hamburgo: Universität Hamburg. Disponible en: http://ediss.sub.uni-hamburg. de/volltexte/2013/6234/pdf/Dissertation.pdf [consultado el 15 de junio de 2013].

Lacadena García-Gallo, Alfonso (2003), "El corpus glífico de Ek' Balam, Yucatán, México”, informe de investigación presentado a la Foundation for the Advancement of Mesoamerican Studies, Inc. Disponible en: http://www.famsi.org/reports [consultado el 10 de abril de 2009].

Lacadena García-Gallo, Alfonso (2004), "Tiempo histórico y tiempo mítico entre los mayas del Periodo Clásico (ss. II-X d.C.)". En Revista de Dialectología y TradicionesPopulares LIX, núm. 1, pp. 83-106. Disponible en: http://rdtp.revistas.csic.es [consultado el 8 de febrero de 2011].

Landa, Diego de (1959[1566]) Relación de las cosas de Yucatan. Introducción por Ángel María Garibay Kintana. México: Porrúa.

Las Casas, Fray Bartolomé de (1967[1536]), Apologética Historia Sumaria, 2 t. México: UNAM.

Malinowski, Bronisław (1948), "Myth in Primitive Psychology". En Robert Redfield (comp.), Magic, Science and Religion and Other Essays by Bronistaw Malinowski. Glencoe: Free Press, pp. 72-124.

Mancini, Silvia (2007), "Comparativism in the history of religions: Some models and key issues". En Religion, núm. 37, pp. 282-293. Disponible en: http://www. elsevier.com/locate/religion [consultado el 13 de agosto de 2008].

Massenzio, Marcello (2005), "The Italian school of 'history of religions"'. En Religion, núm. 35, pp. 209222. Disponible en: http://www.elsevier.com/locate/ religion [consultado el 19 de julio de 2008].

Mendieta, Gerónimo de (1997[1595/96-1604]), Historia eclesiástica indiana, 2 t. Noticias del autor y de la obra por Joaquín García Icazbalceta, estudio preliminar Antonio Rubial García. México: CONACULTA.

Miram, Helga-Maria (1983), Numeral Classifiers im Yukatekischen Maya. Hannover: Verlag für Ethnologie.

Prager, Christian M. (2003), Zahlenklassifikatoren in Hieroglyphen-Inschriften der klassischen Mayakultur:
Beobachtungenzu T87 (Wayeb Note 2). Bélgica: Wayeb, European Association of Mayanists.

Puech, Henri-Charles (1958), "Gnosis and Time" [1951] En Man and Time. Papers from the Eranos Yearbooks. Londres, Routledge \& Keagan Paul, pp. 38-84.

Sabbatucci, Dario (1988), "Kultur und Religion". En Hubert Cancik et al. (ed.), Handbuch religionswissenschaftlicher Grundbegriffe, t. I. Stuttgart: W. Kohlhammer, pp. 43-58.

Rice, Prudence M. (2004), Maya Political Science: Time Astronomy, and the Cosmos. Austin: University of Texas. Rice, Prudence M. (2008), "Time, Power, and the Maya" en Latin American Antiquity, vol. 19, núm. 3, pp. 275-298. Sahagún, Bernardino de (1999[1577]), Historia general de las cosas de Nueva España. Edición de Ángel María Garibay Kintana. México: Porrúa.

Seler, Eduard Georg (1900), Das Tonalamatl der Aubin'schen Sammlung. Eine altmexikanische Bilderhandschrift der Bibliotheque Nationale in Paris (Manuscrits Mexicains Nr. 18-19), 3 t. Berlin: Unger.

Spiro, Melford E. (1966), "Religion: Problems of Definition and Explanation". En Michael Banton (ed.), Anthropological Approaches to the Study of Religion. Londres: Tavistock, pp. 85-126.

Suárez, Jorge A. (1995), Las lenguas indígenas mesoamericanas. México: Instituto Nacional Indigenista, Centro de Investigaciones y Estudios Superiores en Antropología Social.

Taube, Karl A. (1992), The Mayor Gods of Ancient Yucatan. Washington: Dumbarton Oaks Research Library and Collection.

Thompson, J. Eric S. (1971), Maya Hieroglyphic Writing: An Introduction. Norman: University of Oklahoma.

Thompson, J. Eric S. (1972), A Commentary on the Dresden Codex-A Maya Hieroglyphic Book. Philadelphia: American Philosophical Society.

Zimmerman, Charlotte (1963), "The Cult of the Holy Cross: An Analysis of Cosmology and Catholicism in Quintana Roo". En History of Religions, vol. 3, núm. 1, pp. 50-71.

Zimmerman, Charlotte (1965), "The Hermeneutics of the Maya Cult of the Holy Cross”. En Numen, vol. 12, núm. 2, pp. 139-159. 
Figura 1. Los números 1,2 y 3 en su forma antropomorfa
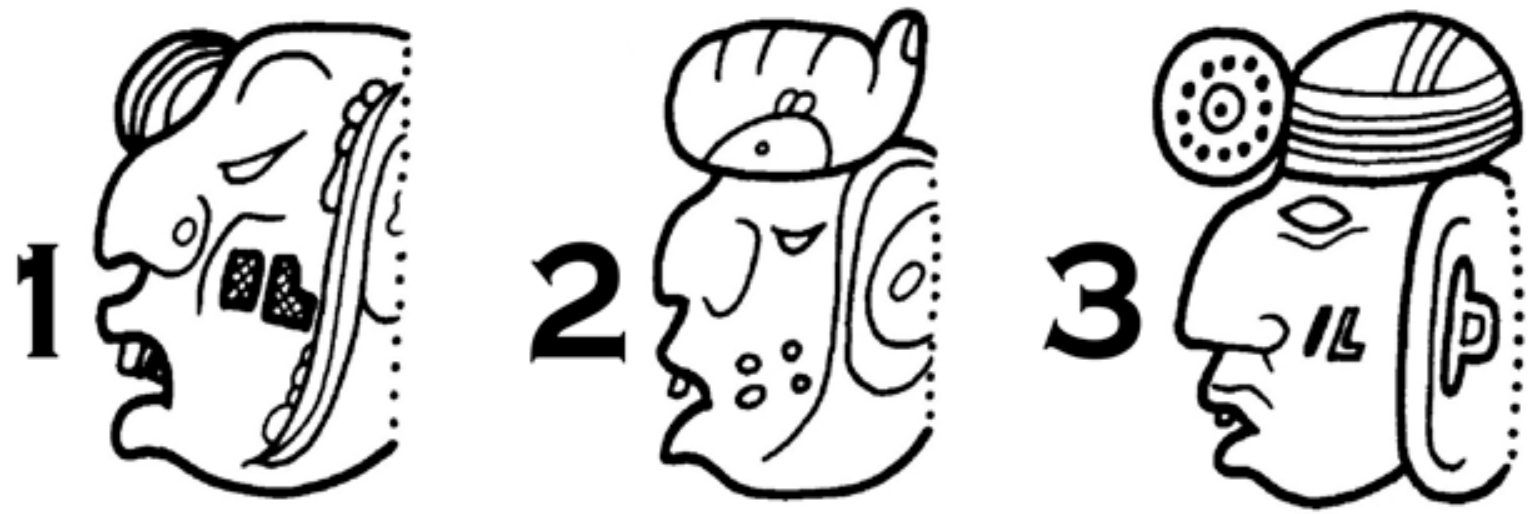

Fuente: dibujo del autor.

Figura 2. El Tzolkin. Códice de Dresde, páginas 5 y 6, registro b
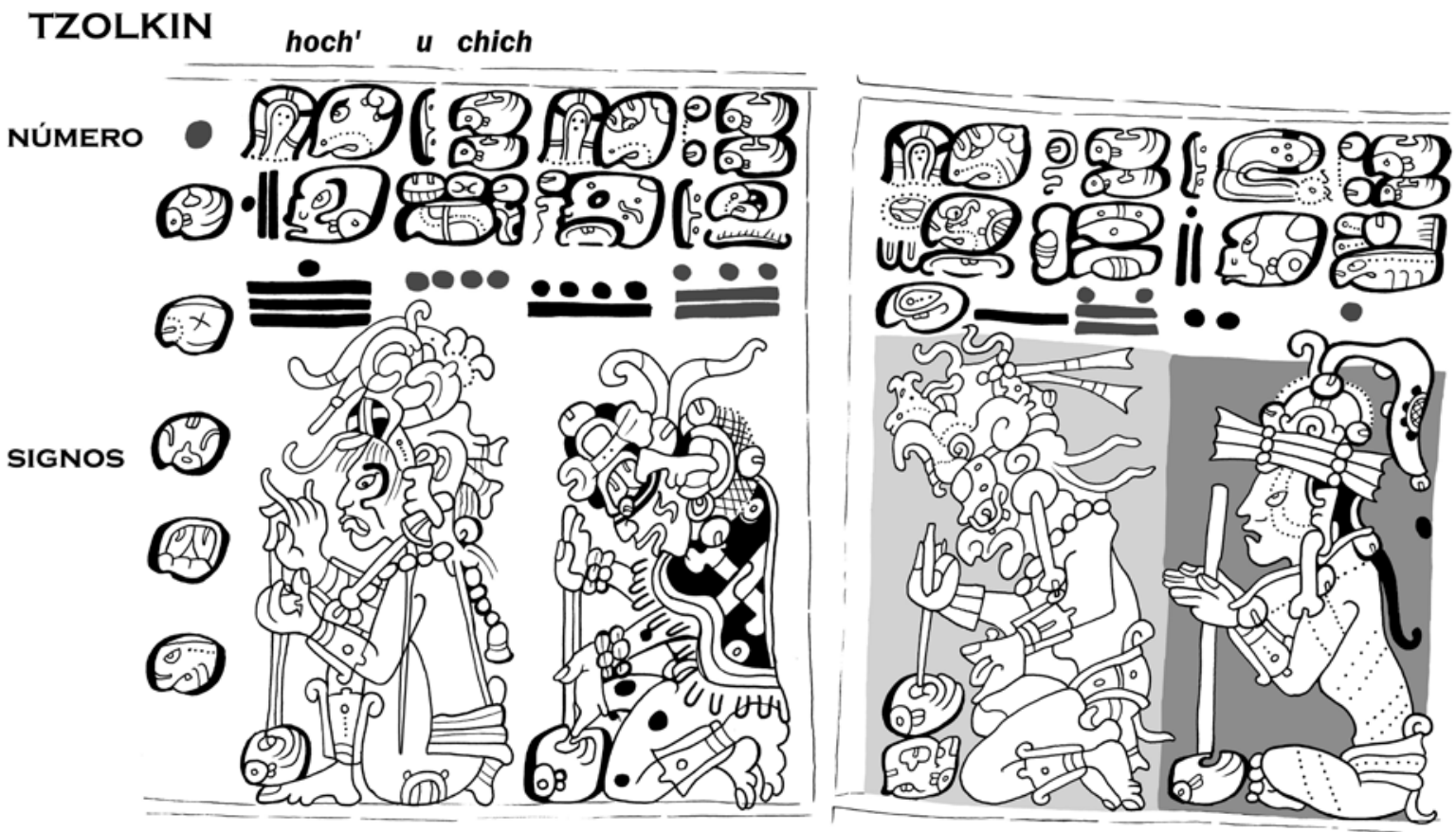

Fuente: dibujo del autor. 
Figura 3. La Rueda Calendárica, la combinación de Tzolkin y Haab. Laxtunich, tablero 2, Chiapas, México

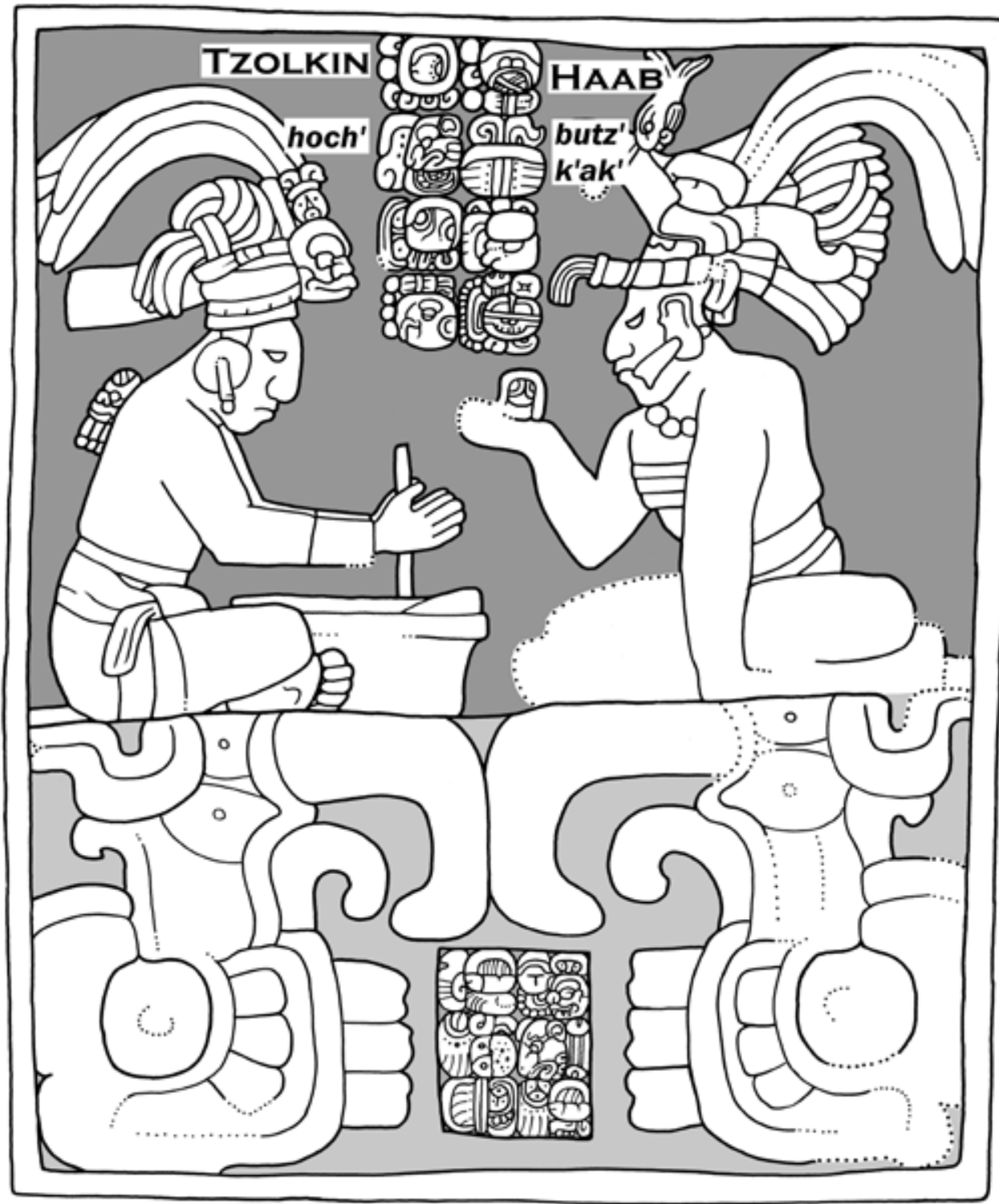

Fuente: dibujo del autor. 
Figura 4. La Cuenta Larga. Dintel del Templo de la Serie Inicial, Estructura $5 C_{4}$, Chichen Itza, Yucatán, México

\section{BAKTUN}
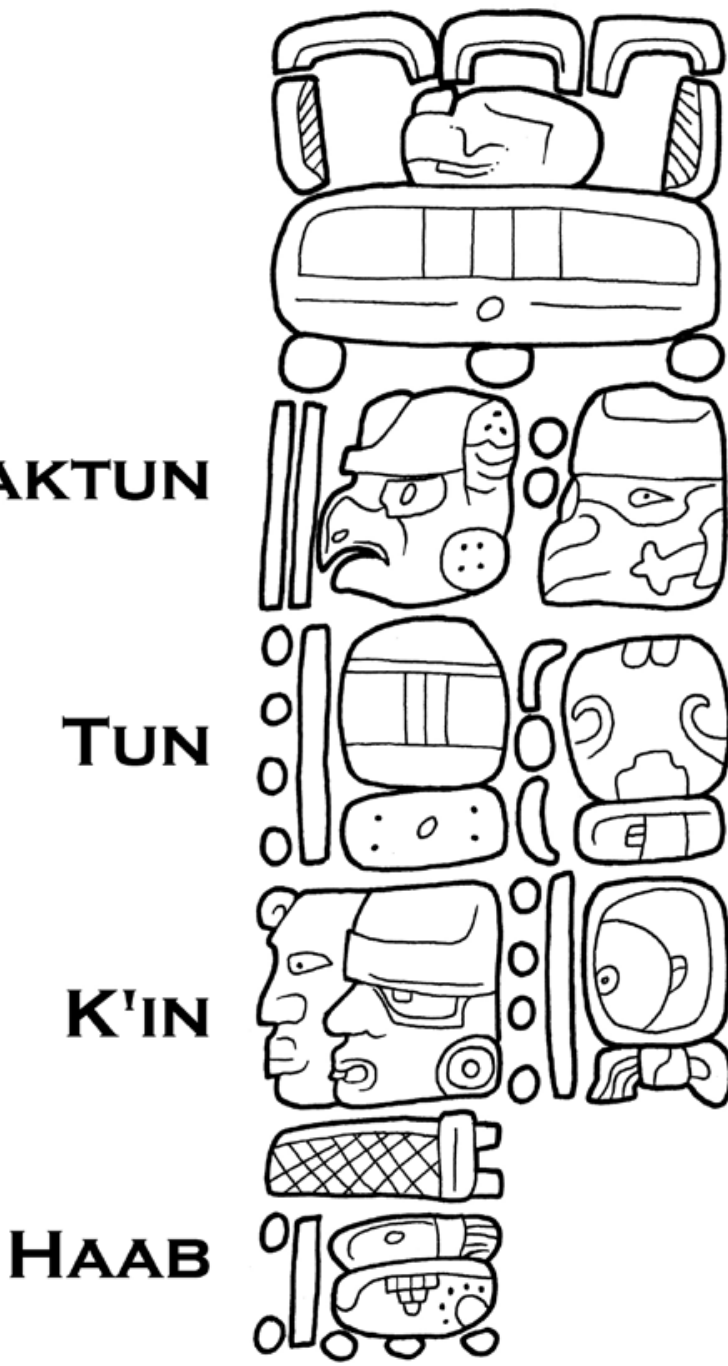

Fuente: dibujo del autor.
K'ATUN

WINAL

TZOLKIN 
Figura 5. El Número de Distancia introducido mediante el verbo tz'ak. Tablero del Palacio, bloques E15-E18, Palenque, Chiapas, México

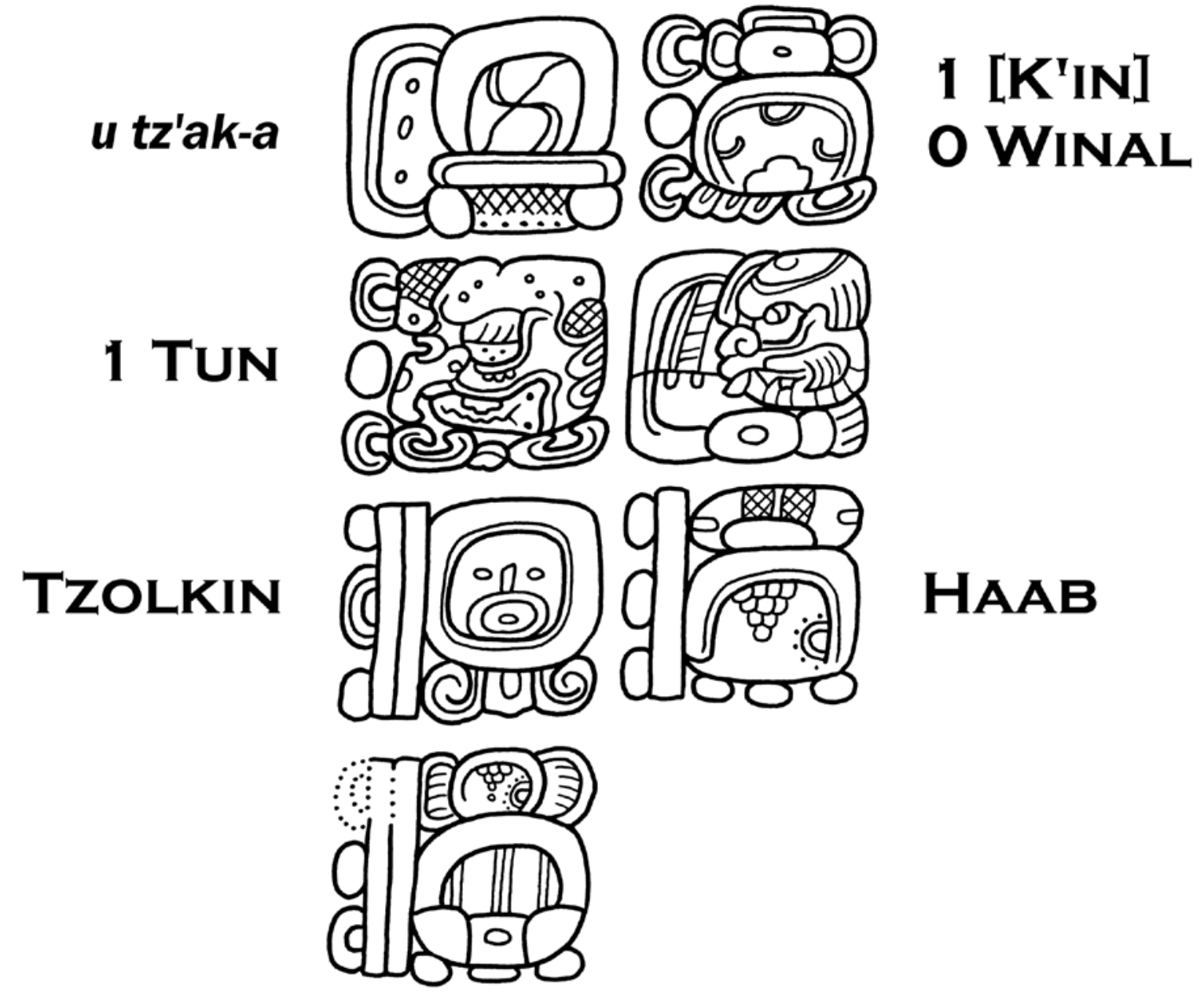

Fuente: dibujo del autor. 
Figura 6. El vocablo te como Clasificador Numeral en la Cuenta Larga y el Haab. Monumento 137, bloques A-I, Toniná, Chiapas, México

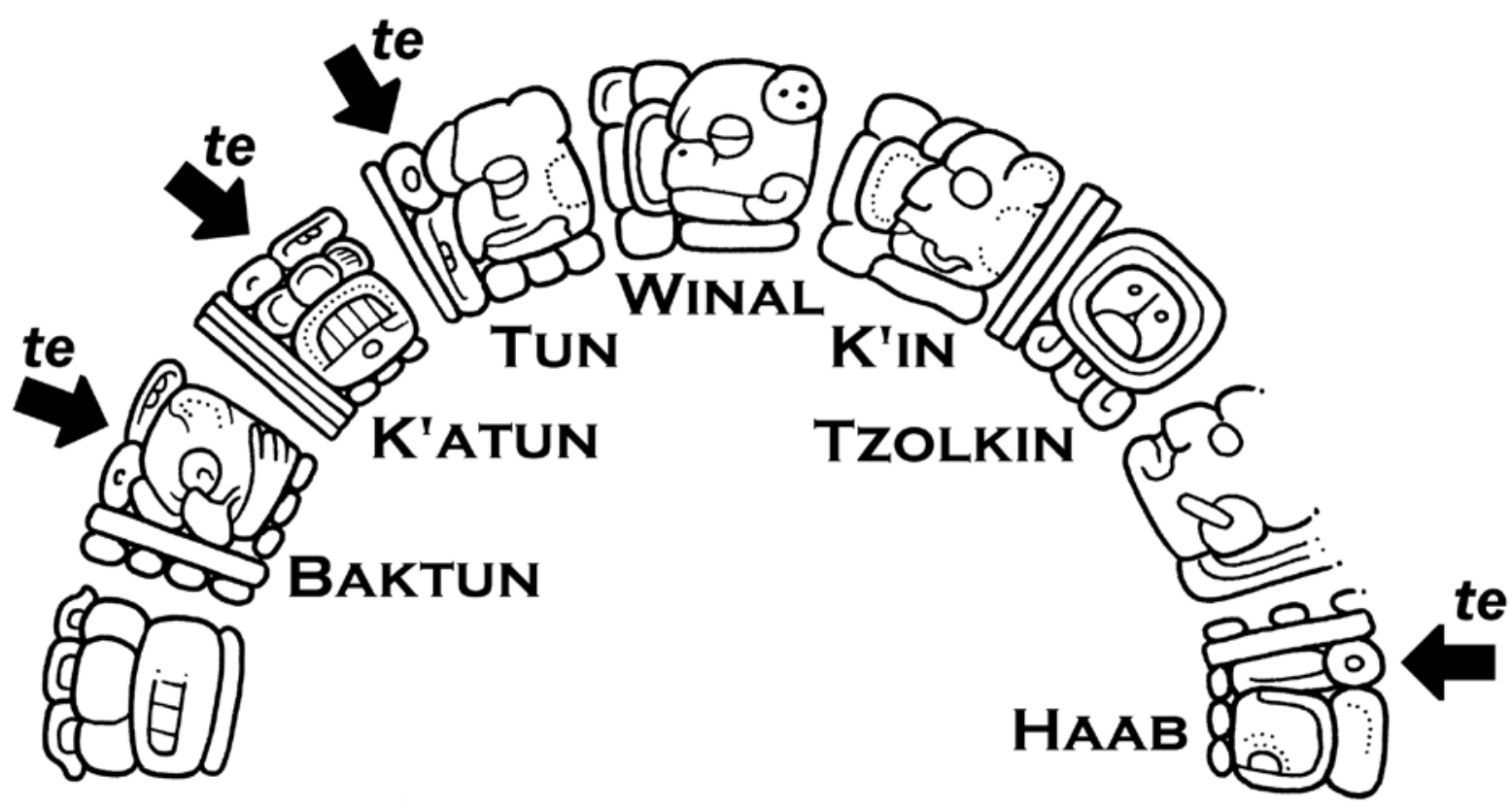

Fuente: dibujo del autor.

Figura 7. El vocablo tak como Clasificador Numeral en la Cuenta Larga. Panel 2, bloque A7, Xcalumkin, Campeche, México

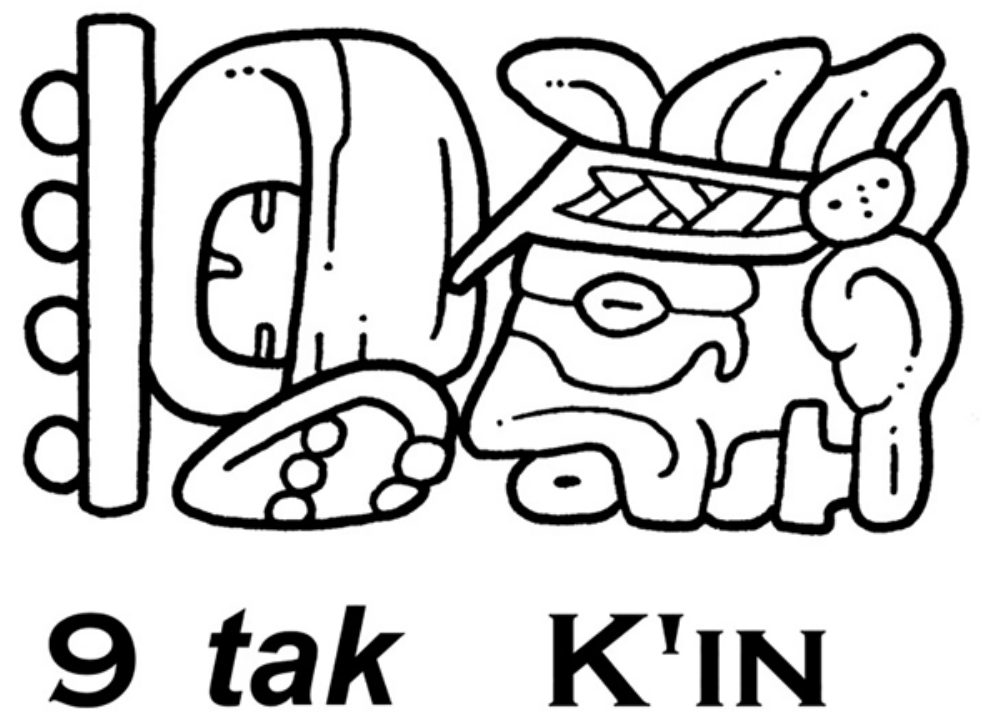

Fuente: dibujo del autor. 